\title{
Alternating bursts of low energy ions and electrons near the substorm onset
}

\author{
T. V. Kozelova ${ }^{1}$, L. L. Lazutin ${ }^{2}$, B. V. Kozelov ${ }^{1}$, N. Meredith $^{3}$, and M. A. Danielides ${ }^{4}$ \\ ${ }^{1}$ Polar Geophysical Institute, Apatity, Murmansk region, 184209, Russia \\ ${ }^{2}$ Russia Space Science Division, Scobeltsyn Institute for Nuclear Physics of Moscow State University, Russia \\ ${ }^{3}$ Mullard Space Science Laboratory, University College, London, UK \\ ${ }^{4}$ Space Physics Group, University of Oulu, Finland
}

Received: 1 February 2006 - Revised: 12 May 2006 - Accepted: 24 May 2006 - Published: 9 August 2006

\begin{abstract}
The substorm associated behavior of the low energy particles $(30 \mathrm{eV}-28.5 \mathrm{keV})$ near the earthward edge of the plasma sheet is examined using data from CRRES during the late growth and early expansion phases of a substorm on 12 March 1991 and their significance for the substorm onset mechanism is discussed. In this substorm, the CRRES was located on $L \sim 6.3$ and $\sim 20^{\circ}$ westward of the substorm onset and observed the sequence of the alternating bursts of the low energy ions and electrons. The bursts of the $0.633-$ $9.6 \mathrm{keV}$ ions occurred 1-2 min before the (7.31-21.7 keV) electron bursts. The first ion burst happened $2 \mathrm{~min}$ before the substorm onset, at the moment of weak brightening of the most equatorial pre-breakup arc near the latitude $\sim 62^{\circ}$. The alternation of the ion and electron bursts may be a signature of a drift-Alfvén ballooning instability on the inner edge of the plasma sheet near substorm onset.
\end{abstract}

Keywords. Magnetospheric physics (Auroral phenomena; Energetic particles, precipitating; Magnetospheric configuration and dynamics)

\section{Introduction}

Injections of energetic particles and dipolarization of the magnetic field are well-known signatures of the magnetospheric substorm in the near-Earth region of the plasma sheet. Dipolarization has been interpreted as the reduction of a portion of the cross-tail current (current disruption) and the formation of a substorm current wedge (McPherron et al., 1973). Two basic models of substorm onset have been suggested: i) the near-Earth current disruption (NECD) in the region of the inner plasma sheet at the distance $\leq 10 R_{E}$ (e.g. Lui, 1991) and ii) the near-Earth neutral line (NENL) at the distance of 15-20 $R_{E}$ (e.g. Baker et al., 1996).

Correspondence to: T. V. Kozelova

(kozelova@pgi.kolasc.net.ru)
From the ground-based observations, the substorm expansion phase onset is recognized by the sharp decrease of the $\mathrm{X}$ component in magnetometer records near the magnetic midnight and by the breakup of the most equatorial arc. It was found in some events that the source region of the arc in the magnetosphere is located in the inner part of the plasma sheet, which is situated earthward of the open-closed field line boundary (Samson et al., 1992; Mende et al., 2003; Dubyagin et al., 2003; Yago et al., 2005). Using various global magnetic field models, the locations of the auroral onset were mapped to equatorial distances in the range of 5-8 $R_{E}$ (Galperin and Feldstein, 1991; Lopez et al., 1991; Frank and Sigwarth, 2000; Dubyagin et al., 2003). On the other hand, in order to identify the onset region in the magnetosphere, auroral particles associated with substorm onset were investigated.

The breakup location relative to the different precipitation boundaries was found using the low altitude satellite observations:

1. The pre-breakup arc near 20-24 MLT is found to correspond to the structure of precipitated electrons with the narrow energy peak of $1-2 \mathrm{keV}$ or of $\sim 3 \mathrm{keV}$ (Dubyagin et al., 2003; Yago et al., 2005).

2. This structure of precipitated electrons with the narrow energy peak is located near the equatorial edge of the diffuse electron precipitation region (Dubyagin et al., 2003; Yago et al., 2005). In this equatorward portion of the auroral precipitation, the average energy of the electrons $E_{e}$ increases towards a higher $\lambda$ - latitude, $d E_{e} / d \lambda>0$ (Newell et al., 1996a, b).

3. This electron precipitation structure, which corresponds to a brightening arc, is located about $0.4-1$ degree poleward of the greatest ion $(0.01-24 \mathrm{keV})$ energy flux location (Dubyagin et al., 2003; Yago et al., 2005). The ion flux maximum matches the boundary "b2i" (Newell et

Published by Copernicus GmbH on behalf of the European Geosciences Union. 


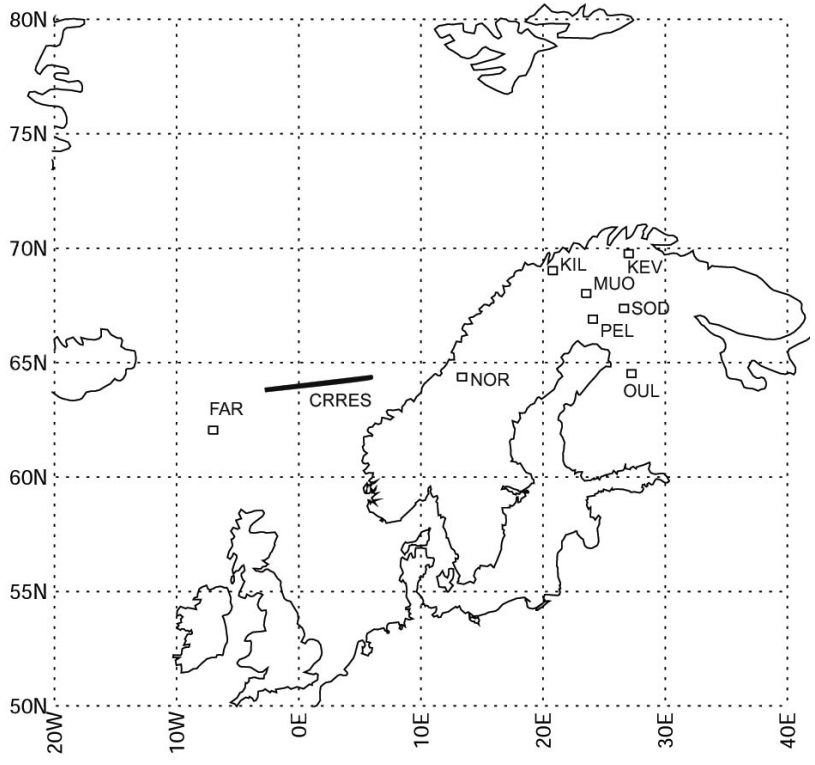

Fig. 1. Locations of ground-based magnetometers and the CRRES mapping to the ionosphere at 1-h interval, 20:00-21:00 UT, during the substorm on 12 March 1991.

al., 1996a, b) or the isotropy boundary (Sergeev et al., 1993). The temperature and energy flux of the ions in the range $3 \mathrm{keV}$ to $30 \mathrm{keV}$ increase with declining latitude (Newell et al., 1996a, b).

Thus, the location of the brightened arc mapped to the equatorial plane is expected to correspond to a region in the magnetosphere where (a) the average energy of the electrons $E_{e}$ increases and (b) the ion energy density rapidly decreases with increasing radial distances. The feature (a) is commonly interpreted as a characteristic of the Alfvén convective boundary for hot electrons (on the inner edge of the plasma sheet). The feature (b) indicates the outer boundary of the trapped energetic ions. The motions of these particle boundaries are observed most frequently during the substorm at geosynchronous orbit in the region near midnight (Kaye and Kivelson, 1979; Walker et al., 1976; Sauvaud and Winckler, 1980).

On the other hand, the current disruptions during the substorm onsets were recognized at the equatorial distances of 5-10 $R_{E}$ in the magnetosphere (Lui, 1978; Kaufmann, 1987; Kozelova and Sakharov, 1989; Jacquey et al., 1991; Kozelova et al., 1999). Therefore, satellites at geosynchronous distances have, during sufficient magnetospheric activity, a good possibility of registering the particle fluxes and magnetic field variations associated with substorm onset plasma instabilities in the pre-midnight sector. An interchange/ballooning instability appears consistent with the near-geosynchronous observations of the substorm onset (Roux, 1985; Kremser et al., 1988; Samson et al., 1992).
Table 1. Ground stations of this case study.

\begin{tabular}{llcccc}
\hline abbr. & name & $\begin{array}{c}\text { geogr. } \\
\text { lat. }\left({ }^{\circ} \mathrm{N}\right)\end{array}$ & $\begin{array}{c}\text { geogr. } \\
\text { lon. }\left({ }^{\circ} \mathrm{E}\right)\end{array}$ & $\begin{array}{c}\text { CGM } \\
\text { lat. }\left({ }^{\circ} \mathrm{N}\right)\end{array}$ & $\begin{array}{c}\text { CGM } \\
\text { lon. }\left({ }^{\circ} \mathrm{E}\right)\end{array}$ \\
\hline FAR & Faroes & 62.05 & 352.98 & 60.72 & 77.44 \\
KEV & Kevo & 69.76 & 27.01 & 66.32 & 109.24 \\
KIL & Kilpisjärvi & 69.02 & 20.79 & 65.88 & 103.79 \\
MUO & Muonio & 68.02 & 23.53 & 64.72 & 105.22 \\
NOR & Nordli, & 64.37 & 13.36 & 61.50 & 94.92 \\
& Norway & & & & \\
OUL & Oulujärvi & 64.52 & 27.23 & 60.99 & 106.14 \\
PEL & Pello & 66.90 & 24.08 & 63.55 & 104.92 \\
SOD & Sodankylä & 67.37 & 26.63 & 63.92 & 107.26 \\
\hline
\end{tabular}

Using the GEOS-2 satellite observations on $6.6 R_{E}$, Roux (1985) found electric field oscillations and changes in the azimuthal component of the energetic ion pressure gradient during substorm dipolarization. Localized field-aligned current structures were found to coincide with the westward travelling surge (WTS) and discrete auroral form. Kozelova et al. (1986a, b) found in the study of the GEOS-2 data that i) the increase of the energetic electron fluxes, ii) the decrease of the energetic ion fluxes, iii) the sharp change in the azimuthal electric field component from eastward to westward, and iv) the magnetic field dipolarization, are all connected with the western edge of the WTS. Holter et al. (1995) performed spectral and wavelet analyses to characterize the transient oscillations during the substorm. They interpreted the obtained low frequency oscillations as a coupled shear Alfvén-slow magnetosonic mode. These oscillations may be excited by the ballooning instability (Miura et al., 1989; Cheng and Qian, 1994).

The CRRES observations at 5-6.6 $R_{E}$ also support an idea that the drift-Alfvén ballooning instability in the neargeosynchronous plasma sheet is a responsible mechanism for substorm onset and initiation of the substorm current wedge (Maynard et al., 1996; Erickson et al., 2000; Kozelova et al., 2000). Maynard et al. (1996) presented the observation of the electric field reversals that correlated with changes in crosstail currents and particle energies occurring before and during the substorm onset at the inner edge of the plasma sheet at 5-6 $R_{E}$. Erickson et al. (2000) found that the initial wave power is dominantly magnetical field aligned and flows towards the ionosphere during eastward oriented electric field. At this time, the drift wave is coupled with the Alfvén mode. Just before the local substorm dipolarization onset, Kozelova et al. (2000) observed one oscillation with an $\sim 90^{\circ}$ phase shift between $E_{y}$ and $B_{z}$. The observed time lag between $B_{z}$ and $E_{y}$ indicated the even mode standing wave structure with the compressional component. 


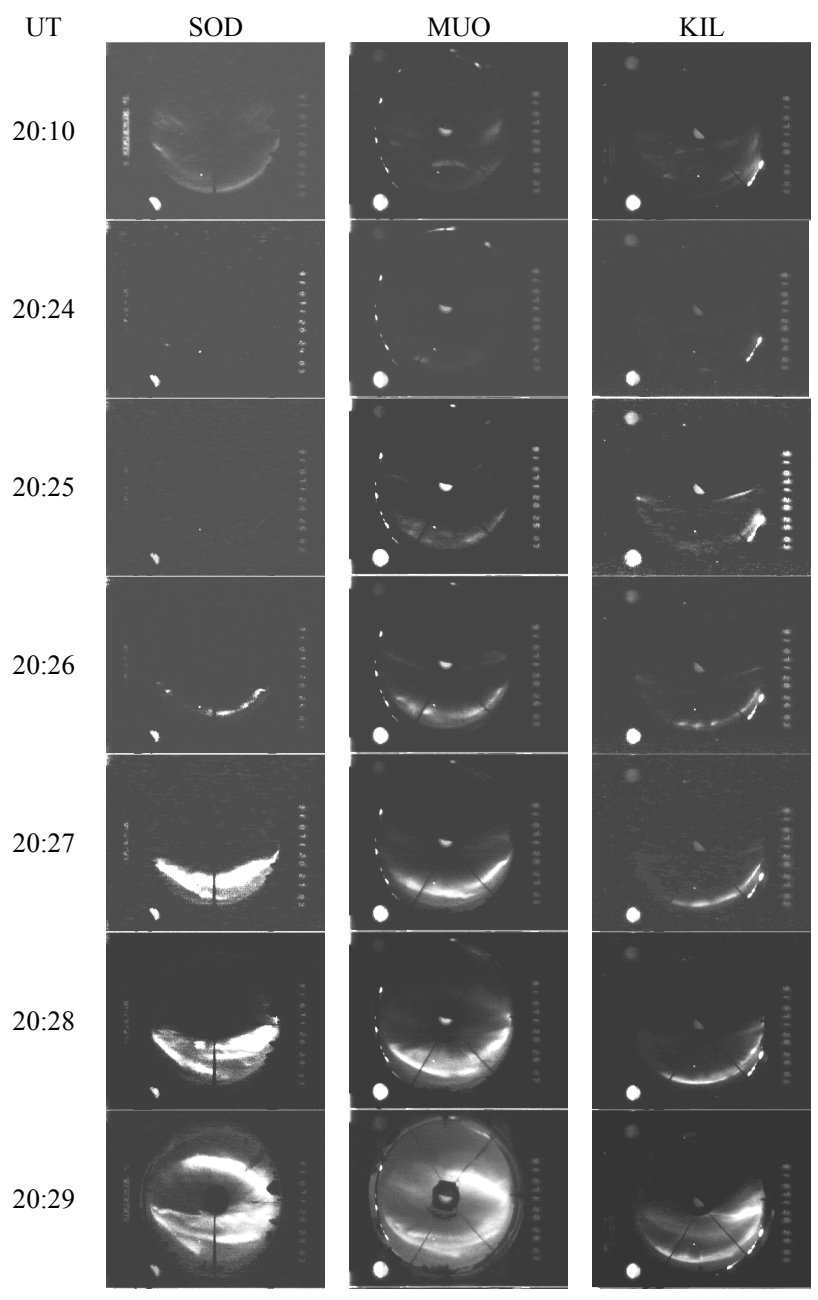

Fig. 2. All-sky camera images taken at SOD, MUO and KIL during the substorm on 12 March 1991. The north (and east) is/are at the top (and right) side of the images.

In this paper, we used data from the CRRES satellite to examine the dynamics of low energy $(100 \mathrm{eV}-30 \mathrm{keV})$ ions and electrons (LEPA data) (Hardy et al., 1993) near the onset of the substorm on 12 March 1991. The CRRES was located on $L \sim 6.3$ (and $\sim 21.5$ MLT) near the earthward edge of the plasma sheet. The CRRES data from the magnetometer (Singer et al., 1992) and from the electron and proton wide-angle spectrometer (EPAS) (Korth et al., 1992) were also used. Ground-based observations include magnetometers and all-sky data from four Finnish stations (SOD, MUO, KIL and KEV). Positions of the ground stations are shown in Table 1. A general structure of this substorm and a particle dynamics were originally reported by Lazutin et al. (2005) from different viewpoints. This substorm is a sequence of optical pseudo-breakups and a breakup above Scandinavia, southward of SOD. The CRRES was located $\sim 20^{\circ}$ westward of the substorm onset epicenter. Figure 1 shows the locations

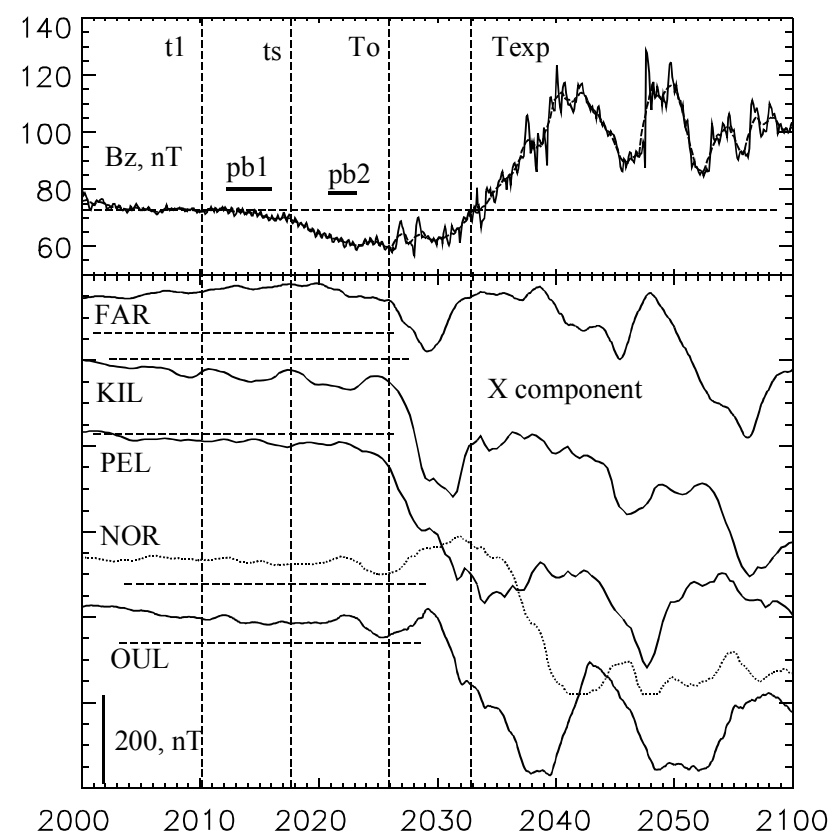

Fig. 3. Substorm on 12 March 1991 . The $B_{z}$ component from the CRRES and $\mathrm{X}$ component from ground magnetometers. Short horizontal solid lines, labeled "pb1" and "pb2", denote weak auroral pseudo-breakups. Horizontal dashed lines are the X component levels during quiet conditions for corresponding magnetometers.

of the magnetometers and the mapping of the CRRES trajectory along the magnetic field line to the ionosphere at the 1-h interval (20:00-21:00) UT during this substorm using the Tsyganenko (1989) model.

\section{Substorm evolution}

The day of 12 March 1991 is characterized by long-lasting ground magnetic activity of 400-800 nT (Lazutin et al., 2005). After 19:50 UT, the southward drifting weak auroral arcs arose near the KIL meridian $\left(\Lambda \sim 117^{\circ}\right)$. Auroral activations are observed in a more poleward region of auroral particle precipitation and may be interpreted as auroral pseudo-breakups.

Figure 2 shows several ASC images borrowed from SOD, MUO, and KIL. At the moment of 20:24 UT, a new, weak arc appears near the latitude $\sim 62^{\circ}$ (equatorward of all arcs observed during growth phase). The optical breakup begins just from this arc at the moment $T_{o}=20: 26$ UT. A large expansion of the substorm begins $7 \mathrm{~min}$ after substorm onset (the moment $T_{\text {exp }}$ ).

Figure 3 presents the $\mathrm{Z}$ component of the magnetic field $\left(B_{z}\right)$, observed by the CRRES, and the magnetometer data at several ground-based stations during the substorm on 12 March 1991. Before $T_{o}$, KIL and PEL were located in the region of the westward electrojet, eastward and poleward of the 


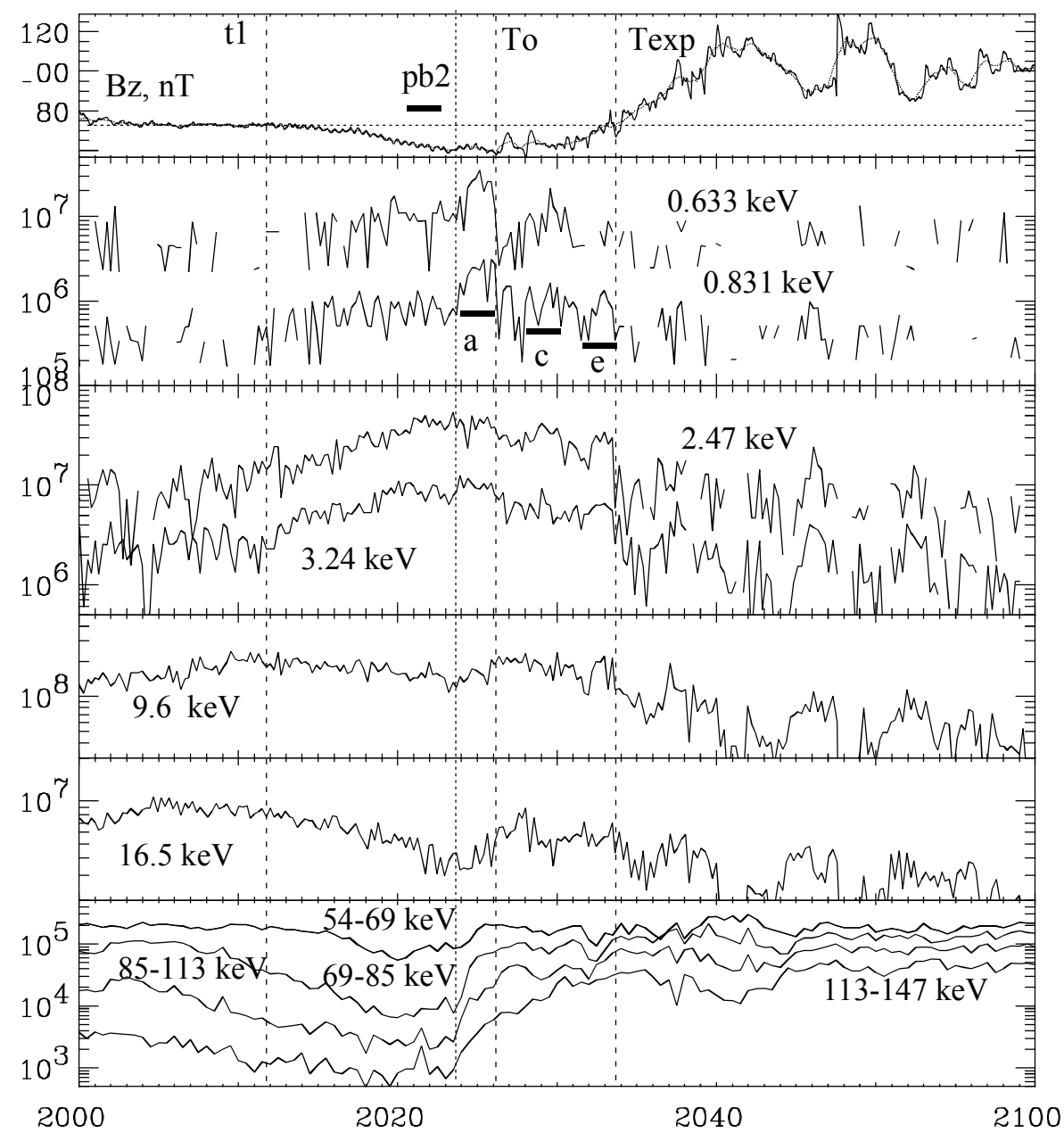

Fig. 4. Substorm on 12 March 1991. Data from the CRRES satellite. From top to bottom: Z-component of the magnetic field; the "A" and "B" populations of LEPA ions with pitch-angles $\sim 90^{\circ}$ are shown in panels (2-3) and (4-5), respectively; flux of high-energy (EPAS) ions with pitch-angles $\sim 90^{\circ}$ are presented in panel 6. Ion flux is in $\mathrm{cm}^{-2} \mathrm{~s}^{-1} \mathrm{sr}^{-1} \mathrm{keV}^{-1}$.

Harang discontinuity, which marks the boundary between the electrojets of the different directions in the late evening sector (Heppner, 1972). At this time, OUL, NOR and FAR were located in the region of the eastward electrojet, equatorward and westward of the Harang discontinuity. From the groundbased observations, a general evolution of this substorm was originally reported by Kozelova et al. (2004).

The first decrease in the X component of the magnetic field at the ground began at 20:22 UT at the station PEL (latitude $\varphi=63^{\circ}$ ). The X component decrease was small until $T_{o}$, when the rate of decrease of the $\mathrm{X}$ component suddenly increased. This was the first substorm intensification, which had a small poleward expansion (to MUO and KIL). Besides, at $T_{o}$, the substorm westward electrojet $J_{W}$ appears suddenly westward of the CRRES on the FAR meridian $\left(\Lambda=80^{\circ}\right)$. However, 3 min later, this substorm intensification recovered, and the global current system with eastward electrojet was reestablished on the FAR. At 20:29-20:30 UT new substorm inten- sification started again near the KIL meridian. This intensification had a significant equatorward expansion (to OUL). The regular westward expansion of the substorm westward electrojet $J_{W}$ was observed after 20:33 UT, during the next intensification, and the equatorward expansion of the magnetic activity began near NOR (Harang discontinuity moved westward). This moment $T_{\exp }=20: 33$ UT was important in the dynamics of the inner magnetosphere.

Thus, before $T_{o}$ CRRES hits the eastward electrojet region and encounters the region 2 field-aligned currents flowing into the ionosphere in the pre-Harang discontinuity sector (Iijima and Potemra, 1978). The westward expansion of the $J_{W}$ can be identified as a transition of the CRRES from the pre-Harang discontinuity to the post-Harang discontinuity sector, where the region 2 field-aligned currents flow from the ionosphere. 


\section{Behavior of the low energy ions}

Figure 4 shows the $\mathrm{Z}$ component of the magnetic field $\left(B_{z}\right)$, LEPA (with 15-s time resolution) and EPAS (with 30-s time resolution) ion data in several channels from the CRRES. In the interval between $t_{1}$ and $T_{\exp }$, the $B_{z}$ component has a slow variation in a hollow form. Besides, some small-scale magnetic field fluctuations appear after $\sim T_{o}$. Usually during the substorm growth phase at near-geosynchronous distances, the $B_{z}$ decrease represents magnetic field line stretching. The $B_{z}$ increase (dipolarization) begins after substorm onset during a substorm expansion phase. We begin our analysis from variations of the low energy ions with the pitch angle $\sim 90^{\circ}$ during a hollow-like magnetic field variation.

\subsection{Slow variation of the ion flux}

During the growth phase stretching of the magnetic field lines on the CRRES (from $t_{1}$ to $T_{o}$ ), the flux of ions with pitch-angles $\sim 90^{\circ}$ was increasing in the energy range from $W_{L}=0.28 \mathrm{keV}$ to $W_{H}=7.31 \mathrm{keV}$. We will name these particles as population " $\mathrm{A}$ ". The ion flux in some energy channels from this population is shown in panels 2 and 3 of Fig. 4. One can see that the flux of $2.47-3.24 \mathrm{keV}$ ions increases earlier than that of the $0.633-0.831 \mathrm{keV}$ ions. This dependence of the enhanced ion flux on energy during the passage into the inner edge of the plasma sheet results in a "nose" distribution in the ion spectrogram (Kerns et al., 1994). The energy $W_{L}$ is a low-cutoff energy below which the drift paths are closed and eastward. The energy $W_{H}$ is a high-cutoff energy above which the drift paths are closed and westward (Kerns et al., 1994). The ions with the energy $W_{L}<W<W_{H}$ convect from the tail to the CRRES position.

During the same interval of time, the flux of ions decreases in the energy range from $9.6 \mathrm{keV}$ to $21.7 \mathrm{keV}$. We will name these particles as population "B". For example, the particle flux for two energy channels from this population is shown in panels 4 and 5 of Fig. 4. One can see that the flux of higher-energy ions from this population begins to decrease earlier than the lower-energy ions. The decrease of the particles flux is usually observed at the outer radiation belt during the growth phase distortion of the magnetosphere to a more tail-like configuration (Walker et al., 1976; Sauvaud and Winckler, 1980).

Thus, different behavior of different energy particles near the inner edge of the plasma sheet during the development of the tail-like magnetic field in the vicinity of the CRRES can separate the distinctive plasma regions and reveal the formation of a sharp transition between these regions. The "A" population is probably associated with the inner boundary of the plasma sheet with the outward (tailward) radial gradient of particle flux. The "B" population can be associated with a population of "old" trapped ions from the outer boundary of trapped particles with an inward (earthwards) radial gradient of particle flux. During the growth phase, this transi- tion boundary moves earthwards. After $T_{o}$, population "A" decreases and population "B" increases at the CRRES position. Both populations are decreasing after $T_{\exp }$, when a large-scale dipolarization of the magnetic field occurs, and the transition boundary is moving tailwards.

\subsection{Ion bursts}

We want to pay attention to the low-energy $(0.633-9.6 \mathrm{keV})$ ion bursts that were observed $\sim 100 \mathrm{~s}$ before $T_{o}$ and $T_{\text {exp }}$. These bursts are marked as "a" and "e" in Fig. 4. Besides, one can see a weak burst "c" at nearly 20:29 UT between the bursts "a" and "e". These three ion bursts with the time duration of $\sim 100 \mathrm{~s}$ were observed with a quasi-period of $3.5 \mathrm{~min}$.

The first ion burst coincides with the sudden enhancement of more energetic (54-147 keV) ion fluxes with pitch-angles $\sim 90^{\circ}$. Simultaneously, the pitch-angle distribution of these energetic ions changes rapidly from the cigar form to the trapped one (Lazutin et al., 2005). The energetic ion flux mesurements (EPAS data) in the four energy channels with a time resolution of $30 \mathrm{~s}$ are shown in the lower panel of Fig. 4. For all energy channels (54-147 keV), the flux increase starts at nearly 20:24 UT. However, for the first energy channel (54-69 keV), a more significant increase of the flux appears with a delay of $\sim 30 \mathrm{~s}$, which is comparable to the time resolution of the data. The ion flux enhancement is the largest for the energy channel (69-85 keV). EPAS ion data with a higher time resolution from three detectors (presented in Lazutin et al., 2005) confirm that the ion flux enhancement near 20:24 UT is "nearly dispersionless" or, more precisely, "dispersionless with a time resolution of $30 \mathrm{~s}$ ".

The first low energy ion burst and a sudden energetic ion enhancement occurred $\sim 2 \min$ before $T_{o}$ and coincided with the appearance of the most equatorial weak arc near the latitude $\varphi=\sim 62^{\circ}$ observed roughly $20^{\circ}$ eastward of CRRES

\section{Plasma sheet electron dynamics}

Figure 5 presents differential flux variations in several electron channels of LEPA data with a 30-s time resolution. The dynamics of trapped electrons near the inner edge of the plasma sheet can help in the interpretation of the observations.

\subsection{Slow electron flux variations}

As seen in Fig. 5, electron flux variations depend on the electron energy. Similar to ion flux variations, the electron fluxes, which increase during the growth phase stretching of the magnetic field lines, will be named as population "A".

Before $T_{o}$, the electron flux increases in the energy range from $0.213 \mathrm{keV}$ to $W_{C}=1.88 \mathrm{keV}$. The fluxes in several energy channels from this population "A" are shown in panel 2 of Fig. 5. One can see that at the CRRES the lowest energy 


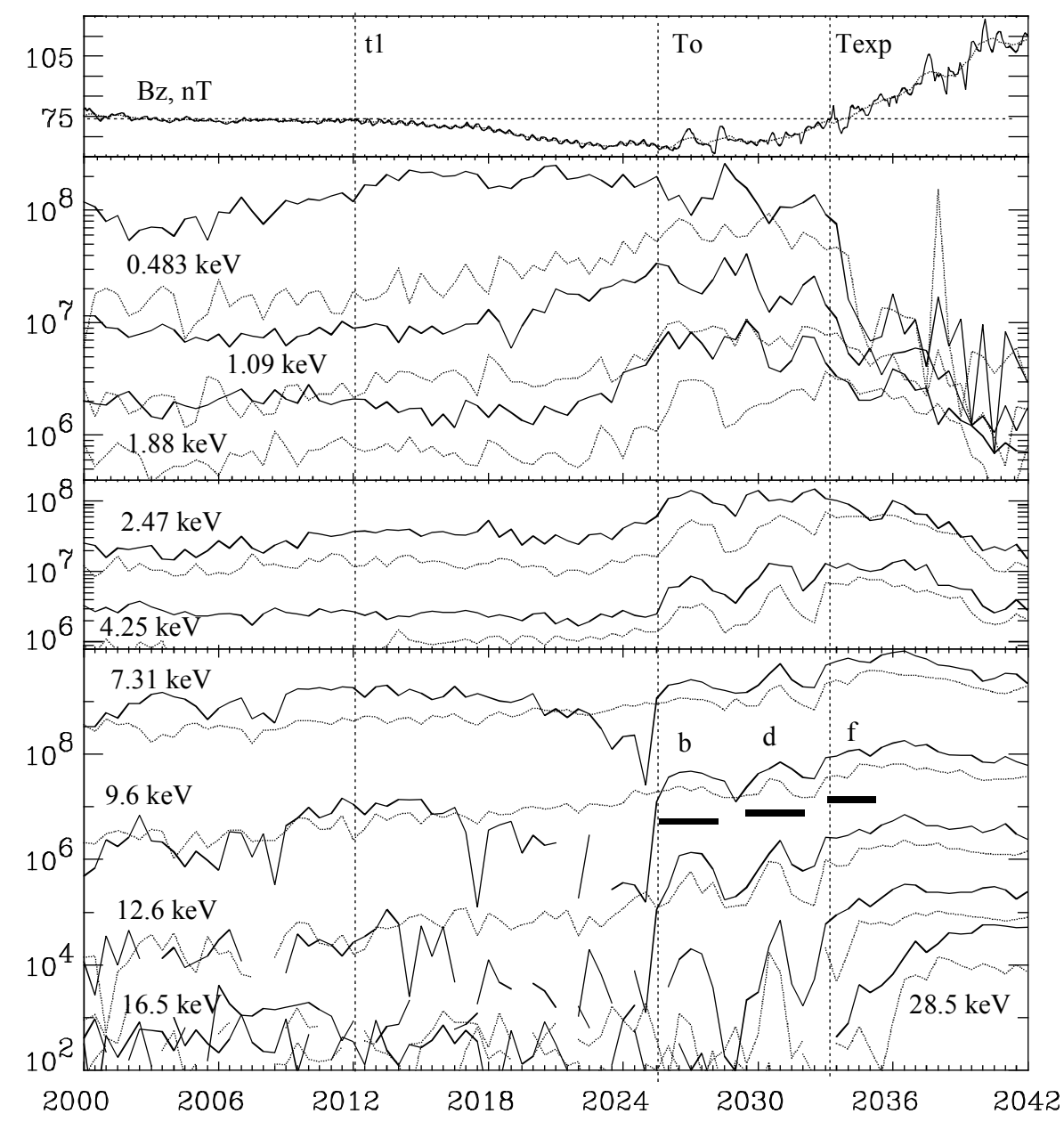

Fig. 5. Substorm on 12 March 1991. Data from the CRRES satellite. From top to bottom: Z-component of the magnetic field; the "A" and "B" populations of LEPA electrons are shown in the panels (2-3) and (4), respectively. Electron flux in $\mathrm{cm}^{-2} \mathrm{~s}^{-1} \mathrm{sr}^{-1} \mathrm{keV}^{-1}$. Solid lines are the flux of particles with pitch-angles $\sim 90^{\circ}$; dashes lines are the flux of particles with pitch-angles near the field-aligned direction.

electrons were observed first. The cutoff energy $W_{C}$ is considered transition energy between the open and closed drift trajectories of the electrons (Kerns et al., 1994). Just before $T_{o}$, the electrons with $W \leq W_{C}$ convect toward the CRRES position from the tail.

During the same time interval before $T_{o}$, the perpendicular flux of electrons with energies $W>W_{C}$ is almost constant or is decreasing. The electron flux is almost constant in the energy range from $2.47 \mathrm{keV}$ to $5.57 \mathrm{keV}$ (panel 3 of Fig. 5). The electron flux decreases in the energy range from $7.31 \mathrm{keV}$ to $16.5 \mathrm{keV}$ (panel 4 of Fig. 5).

According to Kerns et al. (1994), electrons with $W>W_{C}$ move by closed drift paths. The electron fluxes, which decrease or are almost constant during the growth phase, will be named "B" population. We observe in panel 4 of Fig. 5 that the perpendicular flux of the higher-energy $(12.6-16.5 \mathrm{keV})$ electrons in this population begins to decrease earlier than the flux of the lower-energy $(7.31-9.6 \mathrm{keV})$ particles similar to the behavior of particles on the outer boundary of the "old" trapped particles during the inward motion of the boundary (Walker et al., 1976; Sauvaud and Winckler, 1980).

Before $T_{o}$, for the electron energy interval (7.31-16.5 keV) the flux exhibits the minimum for pitch angles around $90^{\circ}$. After $T_{\text {exp }}$, "A" population is decreased and "B" population is increased.

\subsection{Injections of electrons}

At $\sim T_{o}$, the electron flux in four adjacent energy channels in the range (7.31-16.5 keV) grows sharply and exceeds the level which it had before the beginning of the growth phase stretching of the magnetic field lines. One could call these increases "injections" of electrons in a small range of electron energy. The first electron injection begins $\sim 40$ s before significant increases of the $\mathrm{Z}$ component of the magnetic field. The second injection of $21.7-28.5 \mathrm{keV}$ electrons occurrs at $\sim T_{\text {exp }}$, during the substorm expansion phase. 


\subsection{Electron bursts}

During the interval between $T_{o}$ and $T_{\text {exp }}$, one can see two electron bursts, marked "b" and "d" in Fig. 5. Their time duration was $\sim 100 \mathrm{~s}$. Note the third burst, "f", with an indistinct duration, masked by the electron injection. The bursts had little increases of both perpendicular and parallel fluxes of electrons with energies of $2.47-5.57 \mathrm{keV}$ (panel 3 of Fig. 5), which are close to the cut-off energy $W_{C}$. More intensive electron bursts were observed in the higher-energy channels of the "B" population, where the electron flux was decreasing during the substorm growth phase (the bottom panel of Fig. 5). After the first burst, the electron flux in the range (7.31-12.6 keV) exceeds the level which it had before the beginning of the substorm growth phase, after the second burst - in the range (16.5-21.7) keV. After the third burst, the flux of electrons with energies $>28.5 \mathrm{keV}$ exceeds its initial value. The bursts occurred at the leading edge of electron injections. During these electron (2.47-12.6 keV) bursts, the flux of electrons (of " $\mathrm{A}$ " population) with energies of 0.213-1.88 keV decreases (panel 1 of Fig. 5).

\subsection{Parallel fluxes of electrons}

Before $T_{o}$, the parallel flux $J \|$ (dashed lines, Fig. 5) of population "A" electrons with $W \leq 1.88 \mathrm{keV}$ was slowly increasing, similar to the perpendicular fluxes. As the satellite crosses the inner edge of the plasma sheet, the average energy of the electrons, $E_{e}$, is increasing with the increase of the radial distance, $r$, so that $d E_{e} / d r>0$. These fluxes $J \|$ may be associated with the equatorial edge of the diffuse auroral electron precipitation in the ionosphere, where the average energy of $E_{e}$ is increasing with the latitude $\lambda$, providing $d E_{e} / d \lambda>0$ (Newell et al., 1996a,b).

\section{Alternation of ion and electron bursts}

Now it is possible to compare the development of ion and electron bursts and simultaneous variations of the magnetic field at the CRRES during the substorm on 12 March 1991 (Fig. 6). Panel 1 of Fig. 6 presents the sliding 60-s smoothed values of the magnetic field $\mathrm{Z}$ component, $\left\langle B_{z}\right\rangle$, to demonstrate slow changes in the field. Fast fluctuations of the total magnetic field $d B_{t}=B_{t}-<B_{t}>$ are shown in the next panel. Panels 3 and 4 present sliding 45-s smoothed values of the ion fluxes for several channels. Panels 5 and 6 present electron fluxes of several energy channels as in Fig. 5.

From Fig. 6, one can see that the ion bursts occur 1-2 min before the electron bursts. The ion bursts occurred without significant fluctuations in $d B_{t}$, and a dependence of the ion bursts on changes in $\left\langle B_{z}\right\rangle$ was not observed. The electron bursts occurred during small local dipolarizations, with the accompanying increase of the magnetic field fluctuations, with a quasi-period of $\sim 40-60 \mathrm{~s}$.

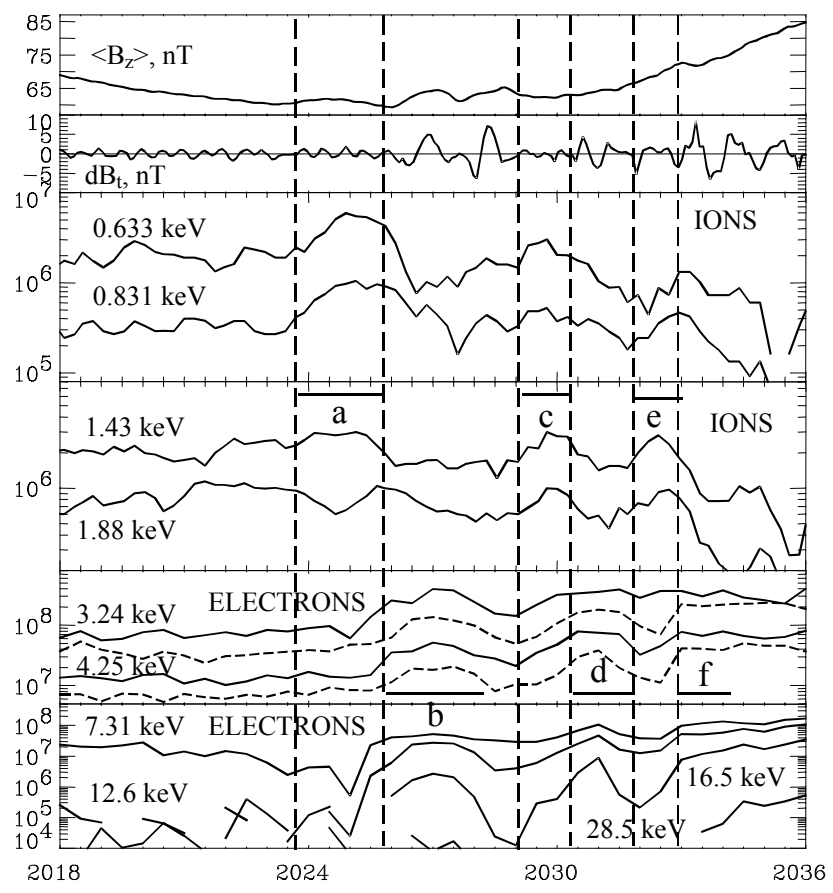

Fig. 6. The alternation of the low-energy ion and electron bursts during the substorm on 12 March 1991.

The electric field measurements (with 30-s time resolution) during this substorm were presented earlier in Lazutin et al. (2005). Before $T_{o}$, the electric field was very small. Impulsive enhancements in the electric field were detected, starting at 20:26 UT (Lazutin et al., 2005). After 20:31 UT, the CRRES electric field cannot be used due to the spacecraft potential becoming too large and driving the sensor's preamplifiers into saturation. During the interval 20:26-20:31 UT, the electric field is nearly zero (in average) and has variations with a period of $\sim 2 \mathrm{~min}$. We cannot confidently separate the variations with the period of $\sim 2 \mathrm{~min}$ in particle flux data; therefore, we don't present the electric field data here.

Lower-frequency oscillations with a period of $\sim 3-4 \mathrm{~min}$, which we observe for particle bursts, are also not seen in electric field. If these oscillations are the same long-period oscillations as in Holter et al. (1995), then they have "a second harmonic standing wave structure along the magnetic field line and develop as a coupled shear Alfvén-slow magnetosonic mode" (Holter et al., 1995). The second harmonic standing wave structure has zero value of the azimuthal electric field at the equatorial plane, where the CRRES satellite was located during considered event. This is a possible explanation for the lack of the electric field oscillations.

\section{Discussion}

The CRRES observation of the substorm on 12 March 1991, when the satellite was located near the inner edge of the 


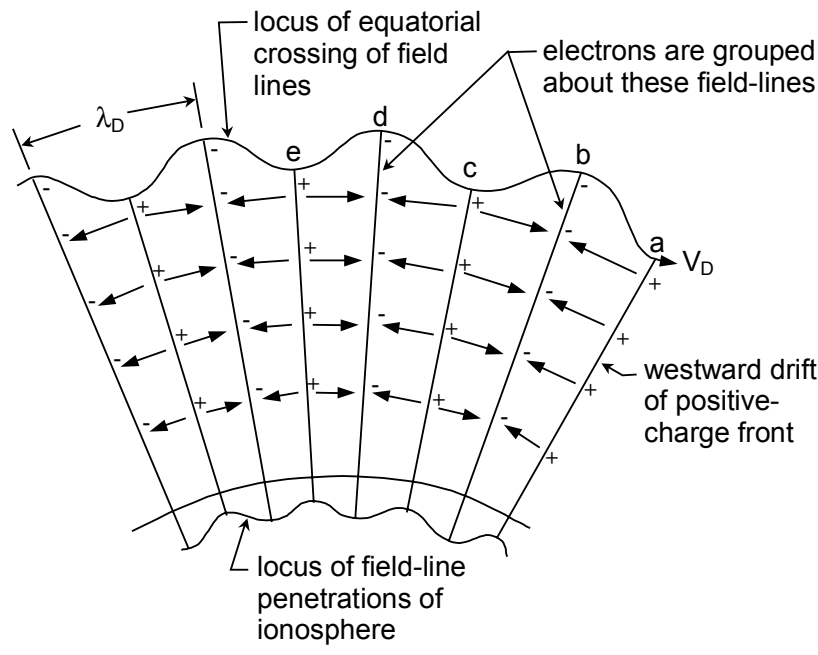

Fig. 7. Illustration of drift-wave resonance of magnetic field lines during azimuthal drift of proton front; adopted from Cladis (1971).

plasma sheet in the pre-midnight sector, supports the nearEarth current disruption (NECD) model of the substorm onset. The sequence of low-energy particle bursts observed at the CRRES may be a signature of an instability of the sharp transition boundary between different particle populations. Below, we discuss and compare our results with other observations.

\subsection{Transition region near the inner edge of the plasma} sheet

It is known that the position of the inner edge of the plasma sheet depends on the cross-tail electric field. Lower-energy electrons come closer to the Earth. When the convection electric field increases (for example, during the substorm growth phase) and/or a satellite is moving across the inner edge of the plasma sheet with the radial tailward gradient of particles, the satellite crosses the boundary for one energy of particles after another and the fluxes of the pertinent electron energies will increase. In our case, the same features characterized population "A", which contained the electrons of the energies $W \leq 1.88 \mathrm{keV}$. But the inward displacement of the drift orbits causes a decrease in the particle flux at the constant energy for the particle population, which has the radial gradient directed toward the Earth, as on the outer boundary of trapped particles (Walker et al., 1976; Sauvaud and Winckler, 1980). In our case, before the $T_{o}$, the flux of ions with energies 9.6-21.7 keV and electrons with the energies $7.31-16.5 \mathrm{keV}$ are decreasing. These trapped particles are "B" population in our notation.

Thus, during the substorm growth phase, the CRRES traverses the magnetosphere region where the average energy of the low-energy $(\leq 1.88 \mathrm{keV})$ electrons is increasing and the density of the ions with $W>9.6 \mathrm{keV}$ is decreasing with growing radial distances. The variations of the parallel fluxes $J \|$ (dashed lines, Fig. 5) of electrons with $W \leq 1.88 \mathrm{keV}$ in population "A" may manifest that the CRRES position is associated with the equatorial edge of the diffuse auroral electron precipitation in the ionosphere. The average energy of the electrons $E_{e}$ is increasing with latitude $\lambda$, so that $d E_{e} / d \lambda>0$ (Newell et al., 1996a, b).

When the tail stretching proceeds during the substorm growth phase, both the inner edge of the plasma sheet and the outer edge of the stable trapped particles steepen and the CRRES observes the formation of the transition boundary between different particle populations with different magnetic field flux tubes at $\sim 6.3 R_{E}$. This boundary may be unstable to westward travelling perturbations (Roux et al., 1991).

The anticorrelation between the ion and electron bursts, found in the investigated substorm on 12 March 1991, is consistent with a schematic development of a drift-Alfvén ballooning instability at the sharp boundary between "dipole" and "tail-like" magnetic field (Roux, 1985; Roux et al., 1991). At the near-geosynchronous distance, the gradient of plasma pressure and the gradient of the magnetic field are oriented in the same direction (earthward). This situation is potentially unstable to the so-called ballooning modes. Energetic ions and electrons, owing to their westward and eastward drifts, respectively, provide charge separation within the perturbated region with the higher plasma pressure. Intense auroral forms and WTS associated with enhanced electron precipitation should map to the position where negative charges tend to prevail (Roux, 1985; Kozelova et al., 1986a,b). This disturbance propagates azimuthally as the drift-Alfvén ballooning wave with $k_{\perp} \gg k_{\|}$. The disturbance may persist for $1-2$, or even only a half of the wave period and then decays. However, a sharp front of energetic ions may support the development of this disturbance.

\subsection{Oscillations associated with a sharp front of energetic ions}

The drift-wave resonance of magnetic field lines may be excited by the azimuthal drift of protons when a sharp gradient of the particle flux occurs in the direction of the gradient- $\boldsymbol{B}$ drift (Cladis, 1971). Cladis (1971) showed that the field lines oscillate as standing waves in the meridional plane and form a wave pattern in the azimuthal direction that moves with the phase velocity equal to the mean azimuthal drift velocity of the particles.

Figure 7 contains a schema of the magnetic field lines' configuration for the drift-wave resonance pattern. This picture is adopted from Fig. 3 of Cladis (1971). In this figure, magnetic field lines that were originally at the same L-shell are shown in their displaced positions (a wave-like line in the equatorial plane, as viewed from above the north pole). According to Cladis (1971), the field lines that are instantaneously at the minimum displacement positions ("a", "c" and "e") contain the maximum positive charge. Those lines 
which are instantaneously at maximum displacement positions ("b", "d", etc.) contain the maximum negative charge.

Now it is possible to compare the sequence of the alternating bursts of the low-energy ions and electrons that are observed during the substorm on 12 March 1991 with the pattern in Fig. 7. A wave-like line on the equatorial plane presents the sharp transition boundary between the regions with different characteristics of plasma and magnetic field. The more dipolar-like magnetic field lines with population "B" are located in the earthward side of this boundary, whereas the tail-like magnetic field lines with population " $A$ " are located in the tailward side of this boundary. The front of the energetic particles is observed at the more tail-like magnetic field configuration, and the maximum of the first burst of electrons occured at more dipolar magnetic field lines. The observed ion bursts "a", "c", and "e" correspond to the regions with the positive charge, and the electron bursts "b", "d", and "f" correspond to the regions with the negative charge. During electron $(2.47-12.6 \mathrm{keV})$ bursts, the flux of electrons with energies of $0.213-1.88 \mathrm{keV}$ (population "A") decreases (panel 1 of Fig. 5). This anticorrelation of the electron fluxes from different populations is consistent with transitions of a boundary between regions with different populations ("A" and "B").

While the flux of the population " $A$ " ions abruptly decreases during the trailing edge of the first burst, the fluxes of the population " $\mathrm{B}$ " ions increase. This behaviour of the particle fluxes is consistent with a transition from a tail-like magnetic field region towards a more dipolar magnetic field region (between the points "a" and "b" at Fig. 7). We can interprete all these transitions as a spatial effect associated with the crossings of the transition boundary. Thus, when the wave structure shown in Fig. 7 is passing the satellite, the satellite observes a sequence of the alternating bursts of the low energy ions and electrons.

During the considered event, the oscillations were stimulated by the dispersionless (with a resolution of $30 \mathrm{~s}$ ) injection of ions with the energy of $54-147 \mathrm{keV}$. This ion injection coincides with the appearance of the most equatorial weak arc, which was observed $\sim 2$ min after the pseudobreakup "pb2" (Fig. 3) and $\sim 2$ min before $T_{o}$ and roughly $20^{\circ}$ eastward of the CRRES. Let us assume that: i) the region of pseudobreakup "pb2" (which occurrs during 20:2120:23 UT) has a westward expansion with a velocity of about $\sim 7.7^{\circ} / \mathrm{min}$, as it was obtained for a substorm expansion in Holter et al. (2004); ii) this velocity is constant during a few minutes; and iii) the CRRES was located $\sim 20^{\circ}$ westward of the "pb2" onset epicenter. Then, the first indication of the approaching pseudo-breakup "pb2" will appear at CRRES at $\sim 20: 23: 30$ UT, nearly simultaneously with the high energy ion injection. Although these moments coincide, the lack of dispersion (or a very small dispersion) during the ion injection is evidence of a local acceleration of the ions.

The beginning of the ground magnetic field disturbance at the meridian of FAR $\left(\sim 15^{\circ}\right.$ westward of the CRRES $)$ that was observed 2 min after the ion injection onset may be associated with the arrival of the westward drifting ions from the CRRES toward the meridian of FAR.

Note that pseudo-breakups in the ionosphere, the appearance of weak magnetic oscillation and the increase of energetic ion flux in the magnetosphere, occurring before $T_{o}$, may testify that the state of the magnetosphere was close to being marginally stable just a few minutes before the moment $T_{o}$.

\subsection{Oscillations with a period of 40-60 s}

During the substorm of 12 March 1991, the higher-frequency oscillations of the magnetic field, with a period of 40-60 s, were observed during the electron bursts. Note that the electron fluxes during the bursts are larger than the initial electron fluxes which occurred before the beginning of the magnetic field line stretching. This fact may indicate that the transition boundary is moving tailward and local acceleration of particles occurs. We suppose that the observed oscillations are probably associated with the kinetic Alfvén waves.

The importance of kinetic Alfvén waves for the acceleration and anomalous transport of the auroral particles was discussed by Hasegawa (1976), Hasegawa and Mima (1978) and Kremser et al. (1988). Kremser et al. (1988) reported on the occurrence of bi-directional field-aligned beams of electrons $(16 \mathrm{keV} \leq E \leq 80 \mathrm{keV})$ that were observed near the substorm onset by the geosynchronous satellite GEOS 2. The beams lasted for about $90 \mathrm{~s}$ (as the bursts) and appeared in the electron pitch angle distributions as the intensity increased at the start of pitch angles of about $20^{\circ}$ (or $160^{\circ}$ ) and peaked at pitch angles of $0^{\circ}$ (or $180^{\circ}$ ). These field-aligned beams were associated with strong increases in the perpendicular electron intensity. Besides, they coincided with strong spatial gradients of the ion intensity and fluctuations of the electric and magnetic fields.

Kremser et al. (1988) considered a model for the beam generation that was based on theoretical investigations by Hasegawa (1976). According to this model, these electron beams may be the result of electron acceleration in the parallel electric field of kinetic Alfvén waves.

We suppose that the electron bursts observed simultaneously with the magnetic field oscillations during the substorm of 12 March 1991 may have a similar origin as the field-aligned electron beams that were observed on GEOS 2 (Kremser et al., 1988). However, in our event, the increase in the flux of field-aligned particles was small. Here it should be noted that before the substorm onset, the CRRES was located in the pre-Harang discontinuity sector, where the region 2 field-aligned currents flow toward the ionosphere. It is possible that conditions in this region were not favorable for a more pronounced evolution of the bursts toward the state of the field-aligned electron beams as in Kremser et al. (1988). 
6.4 Transition between two regimes of transport

The measurements of the bulk velocity in the plasma sheet (Angelopoulos et al., 1992; Borovsky et al., 1998) indicate that there is a great scattering about the mean velocity. This was interpreted as an evidence of the existence of the turbulent transport (Trakhtengerts and Feldstein, 1987; Antonova and Ovchinnikov, 1999).

The presence of the turbulence alters the conductivity and the diffusion properties across the plasma sheet and may permit a cross-scale coupling of localized perturbations into a global perturbation. The transition between the two regimes of transport presents a burst-like process with the characteristic time of $\tau \leq 10 \mathrm{~s}$ and may correspond to auroral activations and substorm onset (Trakhtengerts and Feldstein, 1987, 1991).

In a quasi-steady state, particularly during the growth phase of substorm, the magnetosphere is in a quasi-steady state with a quasi-static electric field driving a quasi-steady earthward convective flow. However, the transient bursts of fast flows were also observed in the plasma sheet (Angelopoulos et al., 1992; Nakamura et al., 1994; Le Contel et al., 2001). The standard convection models, based on constant electrostatic fields, do not describe this regime of flows. Le Contel et al. (2001) showed that the level of the ULF fluctuations at the proton gyrofrequency seems to control the transition between the two regimes of the plasma transport in the near-Earth plasma sheet: a slow transport during the growth phase and a fast transport during the active phase of the substorm.

Impulsive increases (as short lasting bursts) of these waves were regularly observed at the GEOS 2 during the substorm magnetic field dipolarization (Perraut et al., 1993, 2000; Le Contel et al., 2001). These spatially localized waves are related to field-aligned currents, Perraut et al. (2000) called them the current-driven Alfvén (CDA) waves. Bursts of CDA waves coincide with the increases in the flux of electrons with energy $>20 \mathrm{keV}$ at pitch-angles $\sim 90^{\circ}$ and, simultaneously, with the increases of low-energy (2.5-7 keV) electron fluxes at pitch-angles $<20^{\circ}$ (Perraut et al., 1993, 2000). Also, the high-intensity CDA waves may isotropize and heat the ions (Perraut et al., 1998).

We turn our attention to the substorm of 12 March 1991 from these positions. Then the pseudo-breakups and slow variations of the particles and the magnetic field occurred before $T_{o}$ became a signature of convective flow, as well as an instability, which began to develop slowly. The dispersionless enhancement of the energetic $54-147 \mathrm{keV}$ proton fluxes and the sequential sharp increase in the electron flux with the energy of 7.32-12.6 keV may correspond to the transition between the convective (laminar) and turbulent regimes of the plasma flows.

\section{Summary}

In this study we examined the low-energy $(30 \mathrm{eV}-28.5 \mathrm{keV})$ particle flux variations observed by the CRRES near the earthward edge of the plasma sheet at $L \sim 6.3$ and $\sim 20^{\circ}$ westward the substorm onset during the substorm on 12 March 1991. Some of the main results of the presented analysis are as follows:

1. During the growth phase magnetic field stretching, the ion and electron flux variations depend on the particle energies: the flux of low-energy (with $\left.W<W_{c}=1.88 \mathrm{keV}\right)$ electrons and ions $(0.28-7.31 \mathrm{keV})$ is increasing. These particles are convecting toward the CRRES from the tail. The flux of the higher-energy electrons (7.31-16.5 keV) and ions (9.6-21.7 keV) is decreasing. These particles are trapped on the closed drift paths. These changes may imply that both the inner edge of plasma sheet and the outer boundary of higherenergy "old" particles steepen. The transition region between different particle populations of the plasma sheet, dipolar and tail-like flux tubes, narrowed and moved earthwards.

2. The sequence of the alternating bursts of the low-energy ions and electrons begins $2 \mathrm{~min}$ before the substorm onset. The bursts of the ions $(0.633-9.6 \mathrm{keV})$ had a time duration of 100-120 s and a quasi-period of 3-4 min. The ion bursts occurred 1-2 min before the electron $(2.47-21.7 \mathrm{keV})$ bursts. The electron bursts were associated with the small, local increase in the $B_{z}$ component and in the magnetic field fluctuations.

3. The beginning of the sequence of the low-energy particles bursts coincides with the enhancement of the highenergetic (54-147 keV) ion flux. The enhancement of the high-energetic ion flux happened at the moment of a weak brightening of the most equatorial pre-breakup arc near the latitude $\sim 62^{\circ}$.

4. Near the substorm onset, the substorm westward electrojet, which is localized $\sim 20^{\circ}$ westward of the CRRES, increases sharply. Simultaneously, the first injection of electrons was observed in a narrow energy range of $7.31-12.6 \mathrm{keV}$. This first electron injection began $\sim 40 \mathrm{~s}$ before a significant increase in the $\mathrm{Z}$ component of the magnetic field. The injection of higher-energy electrons (12.6-21.7 keV) was observed later, at the beginning of a large substorm expansion.

The variations of the ion and electron bursts may be associated with the multiple crossings of the transition boundary (discussed above) which has an oscillatory behavior. A sharp gradient of energetic ions in the direction of the gradient$\boldsymbol{B}$ drift may excite the drift-wave resonance of the magnetic field lines (Cladis, 1971) and support oscillations on the transition boundary. Our analysis supports the idea about the 
drift-Alfvén ballooning instability near the inner edge of the plasma sheet, as associated with the initiation of a substorm onset.

Acknowledgements. Authors are grateful to A. Korth for energetic particle data and H. Singer for magnetic field data from the CRRES spacecraft. The authors are grateful to observers and scientists responsible for the ground based magnetic and auroral observations (IMAGE, AASI). The authors thank the SAMNET team for magnetometer data; SAMNET is a PPARC National Facility operated by Lancaster University. The work of T. V. Kozelova and B. V. Kozelov is supported by the Division of Physical Sciences of the Russian Academy of Sciences (program DPS-18).

Topical Editor I. A. Daglis thanks two referees for their help in evaluating this paper.

\section{References}

Angelopoulos, V., Baumjohann, W., Kennel, C. F., Coroniti, F. V., Kivelson, M. G., Pellat, R., Walker, R. J., Lühr, H., and Paschmann, G.: Bursty bulk flows in the inner central plasma sheet, J. Geophys. Res., 97, 4027-4039, 1992.

Antonova, E. E. and Ovchinnikov, I. L.: Magnetostatically equilibrated plasma sheet with developed medium scale turbulence: structure and implications for substorm dynamics, J. Geophys. Res., 104, 17 289-17 298, 1999.

Baker, D. N., Pulkkinen, T. I., Angelopoulos, V., Baumjohann, W., and McPherron, R. L.: The neutral line model of substorm: past results and present view, J. Geophys. Res., 101, 12 975-13 010, 1996.

Borovsky, J. E., Thomsen, M. F., and Elphic, R. C.: The driving of the plasma sheet by the solar wind, J. Geophys. Res., 103, 17 617-17 639, 1998.

Cheng C. Z. and Qian, Q.: Theory of ballooning-mirror instabilities for anisotropic pressure plasmas in the magnetosphere, J. Geophys. Res., 99, 11 193-11 209, 1994.

Cladis, J. B.: Multiply coupled oscillations of field lines in the magnetosphere: modulation of trapped particles and ionospheric currents, J. Geophys. Res., 76, 2345-2356, 1971.

Dubyagin, S. V., Sergeev, V. A., Carlson, C. W., Marple, S. R., Pulkkinen, T. I., and Yahnin, A. G.: Evidence of near-Earth breakup location, Geophys. Res. Lett., 30, 1282, doi:10.1029/2002GL016569, 2003.

Erickson, G. M., Maynard, N. C., Wilson, G. R., and Burke, W. J.: Electromagnetics of substorm onsets in the near-geosynchronous plasma sheet, Proc. 5th International Conference on Substorms, St.-Petersburg, Russia, 16-20 May 2000 (ESA SP-443, July 2000), 385-388, 2000.

Frank, L. A. and Sigwarth, J. B.: Findings concerning the positions of substorm onsets with auroral images from the Polar spacecraft, J. Geophys. Res., 105, 12 747-12 761, 2000.

Galperin, Y. I. and Feldstein, Y. I.: Auroral luminosity and its relationship to magnetospheric plasma domains, in: Auroral Physics, edited by: Meng, C. I., Rycroft, M. J., and Frank, L. A., 207-222, Cambridge Univ. Press, New York, 1991.

Hardy, D. A., Walton, D. M., Johnstone, A. D., Smith, M. F., Gough, M. P., Huber, A., Pantazis, J., and Burkhardt, R.: Low Energy Plasma Analyzer, IEEE Trans. Nucl. Sci., 40, 246-251, 1993.
Hasegawa, A. and Mima, K.: Anomalous transport produced by kinetic Alfven waves turbulence, J. Geophys. Res., 83, 11171123, 1978.

Hasegawa, A.: Particle acceleration by MHD surface wave and formation of aurora, J. Geophys. Res., 81, 5083-5090, 1976.

Heppner, J. P.: The Harang discontinuity in auroral belt ionospheric currents, Geofysiske Publikasjoner, 29, 105-120, 1972.

Holter, Ø., Altman, C., Roux, A., Perraut, S., Pedersen, A., Pécseli, H., Lysekk, B., Trulsen, J., Korth, A., and Kremser, G.: Characterization of low frequency oscillations at substorm breakup, J. Geophys. Res., 100, 19 109-19 119, 1995.

Holter, Ø., Galopeau, P., Roux, A., Perraut, S., Pedersen, A., Korth, A., and Bösinger, T.: Two satellite study of substorm expansion near geosynchronous orbit, Ann. Geophys., 22, 4299-4310, 2004.

Iijima, T. and Potemra, T. A.: Large scale charactetistics of fieldaligned currents associated with sibstorms, J. Geophys Res., 83, 599-615, 1978.

Jacquey, C., Sauvaud, J. A., and Dandouras, J.: Location and propagation of the magnetotail current disruption during substorm expansion: analysis and simulation of an ISEE multi-onset event, Geophys. Res. Lett., 18, 389-392, 1991.

Kaufmann, R. L.: Substorm currents: growth phase and onset, J. Geophys Res., 92, 7472-7489, 1987.

Kaye, S. M. and Kivelson, M. G.: Time dependent convection electric fields and plasma injection, J. Geophys. Res., 84, 4183-4188, 1979.

Kerns, K. J., Hardy, D. A., and Gussenhoven, M. S.: Modeling of convection boundaries seen by CRRES in $120-\mathrm{eV}$ to $28-\mathrm{keV}$ particles, J. Geophys. Res., 99, 2403-2414, 1994.

Korth, A., Kremser, G., Wilken, B., Guttler, W., Ullaland, S. L., and Koga, R.: Electron and proton wide-angle spectrometer (EPAS) on the CRRES spacecraft, J. Spacecraft Rockets, 29, 609-614, 1992.

Kozelova, T. V., Lazutin, L. L., Kozelov, B. V., Meredith, N., Danielides, M., Jussila, J., and Korth, A.: Multiple-onset substorm case study: pre-onset, auroral onset and expansion, Proc. 7th International Conference on Substorm, Levi, Lapland, Finland, 21-27 March 2004, 176-179, 2004.

Kozelova, T. V. and Sakharov, Ya. A.: Configuration of the magnetic field in a geostationary orbit during a substorm, Geomagnetism and Aeronomy, 29(2), 213-216, 1989.

Kozelova, T. V., Kozelov, B. V., and Lazutin, L. L.: Substorm large impulsive electric fields observed by CRRES, Proc. 5th International Conference on Substorms, St.-Petersburg, Russia, 16-20 May 2000 (ESA SP-443, July 2000), 393-396, 2000.

Kozelova, T. V., Lazutin, L. L., and Kozelov, B. V.: Dipolarization and the current perturbations in the magnetosphere from the CRRES satellite data, Geomagnetism and Aeronomy (in Russian), 39(1), 15-26, 1999.

Kozelova, T. V., Treilhou, J.-P., Korth, A., Kremser, G., Lazutin, L. L., Melnikov, A., Pedersen, A., and Sakharov, Ya.: The active phase of the substorm from ground based and satellite data, Geomagnetism and Aeronomy (in Russian), 26(6), 963-969, 1986a.

Kozelova, T. V., Pudovkin, M. I., Lazutin, L. L., Pedersen, A., and Glassmeier, K.-H.: The electric field behaviour in the magnetosphere on $6.6 R_{E}$ within active region during the substorm, Geomagnetism and Aeronomy (in Russian), 26(4), 621-627, $1986 \mathrm{~b}$. 
Kremser, G., Korth, A., Ullaland, S. L., Perraut, S., Roux, A., Pedersen, A., Schmidt, R., and Tanskanen, P.: Field-aligned beams of energetic electrons $(16 \mathrm{keV} \leq \mathrm{E} \leq 80 \mathrm{keV})$ observed at geosynchronous orbit at substorm onsets, J. Geophys. Res., 93, 1445314 464, 1988.

Lazutin, L. L., Kozelova, T. V., Danielides, M., Meredith, N., Kozelov, B. V, Jussila, J., and Korth, A.: Aurora, currents and particle dynamics in the inner magnetosphere: case study of multiple-onset substorm of March 12, 1991, Proc. 31st Annual European Meeting on Atmospheric Studies by Optical Methods and 1st International Riometer Workshop, 22 to 28 August 2004, Ambleside, The Lake District, UK, 50-72, 2005.

Lazutin, L. L., Korth, A., and Kozelova, T. V.: Fast bursts of high energy protons and their role in triggering of the substorm onset instability, Proc. Sixth International Conference on Substorm, Seattle, 340-346, 2002.

Le Contel, O., Roux, A., Perraut, S., Pellat, R., Holter, O., Pedersen, A., and Korth, A.: Possible control of plasma transport in the near-Earth plasma sheet via current-driven Alfven waves $\left(\mathrm{f} \cong \mathrm{f}_{H+}\right)$, J. Geophys. Res., 106, 10 817-10 827, 2001.

Lopez, R. E., Spence, H. E., and Meng, C.-I.: DMSP F7 observations of a substorm field-aligned current, J. Geophys. Res., 96, 19409-19415, 1991.

Lui, A. T. Y.: A synthesis of magnetospheric substorm models, J. Geophys. Res., 96, 1849-1856, 1991.

Lui, A. T. Y.: Estimates of current changes in the geomagnetotail associated with a substorm, Geophys. Res. Lett., 5, 853-856, 1978.

Maynard, N. C., Burke, W. J., Basinska, E. M., Erickson, G. M., Hughes, W. J., Singer, H. J., Yahnin, A. G., Hardy, D. A., and Mozer, F. S.: Dynamics of the inner magnetosphere near times of substorm onsets, J. Geophys. Res., 101, 7705-7736, 1996.

McPherron, R. L., Russel, C. T., and Aubry, M. P.: Satellite studies of magnetospheric substorms on August 15, 1968, 9. Phenomenological model for substorms, J. Geophys Res., 78, 31313149, 1973.

Mende, S. B., Carlson, C. W., Frey, H. U., Peticolas, L. M., and Ostgaard, N.: FAST and IMAGE-FUV observations of a substorm onset, J. Geophys. Res., 108(A9), 1344, doi:10.1029/2002JA009787, 2003.

Miura, A., Ohtani, S., and Tamao, T.: Ballooning instability and structure of diamagnetic hydromagnetic waves in a model magnetosphere, J. Geophys. Res., 94, 15 231-15 242, 1989.

Nakamura, T., Baker, D. N., Fairfield, D. H., Mitchell, D. G., McPherron, R. L., and Hones Jr., E. W.: Plasma flow and magnetic field characteristics near the midtail neutral sheet, J. Geophys. Res., 99, 23 591-23 602, 1994.

Newell, P. T., Feldstein, Ya. I., Galperin, Yu. I., and Meng, C.-I.: Morphology of nightside precipitation, J. Geophys. Res., 101, 10737-10 748, 1996a.
Newell, P. T., Feldstein, Ya. I., Galperin, Yu. I., and Meng, C.-I.: Correction to "Morphology of nightside precipitation", J. Geophys. Res., 101, 17 419-17 421, 1996b.

Perraut, S., Le Contel, O., Roux, A., Pellat, R., Kort, A., Holter, O., and Pedersen, A.: Disruption of parallel current at substorm breakup, Geophys. Res. Lett., 27, 4041-4044, 2000.

Perraut, S., Le Contel, O., Roux, A., Pellat, R., Pedersen, A., and Kort, A.: Evidence for a substorm trigger, in: Substorm-4, edited by: Kokubun, S. and Kamide, Y., Astrophysics and space science library, 238, 349-354, 1998.

Perraut, S., Morane, A., Roux, A., Pedersen, A., Schmidt, R., Kort, A., Kremser, G., Aparicio, B., and Pellinen, R.: Characterization of small scale turbulence observed at substorm onsets: relationships with parallel acceleration of particles, Adv. Space Res., 13(4), 217-222, 1993.

Roux, A., Perraut, S., Robert, P., Morane, A., Pedersen, A., Korth, A., Kremser, G., Aparicio, B., Rodger, D., and Pellinen, R.: Plasma sheet instability related to the westward traveling surge, J. Geophys. Res., 96, 17 697-17 714, 1991.

Roux, A.: Generation of field-aligned current structures at substorm onset, Proc. ESA Workshop on Future Missions in Solar, Heliospheric nd Space Plasma Physics, Garnisch-Partenkirchen, Germany, ESA SP-235, 151-159, 1985.

Samson, J. C., Lyons, L. R., Xu, B., Creutzberg, F., and Newell, P.: Proton aurora and substorm intensifications, Geophys. Res. Lett., 19, 2167-2170, 1992.

Sauvaud, J.-A. and Winckler, J. R.: Dynamics of plasma, energetic particles, and fields near synchronous orbit in the nighttime sector during magnetospheric substorms, J. Geophys. Res., 85, 2043-2056, 1980.

Sergeev, V. A., Malkov, M. V., and Mursula, K.: Testing the isotropic boundary algorithm method to evaluate the magnetic field configuration in the tail, J. Geophys. Res., 98, 7609-7620, 1993.

Singer, H. J., Sullivan, W. P., Anderson, P., Mozer, F., Harvey, P., Wygant, J., and McNeil, W.: Fluxgate magnetometer instrument on the CRRES, J. Spacecraft Rockets, 29, 599-601, 1992.

Trakhtengerts, V. Yu. and Feldstein, A. Ya.: Turbulent regime of magnetospheric convection, Geomagnetism and Aeronomy (in Russian), 27, 258-264, 1987.

Trakhtengerts, V. Yu. and Feldstein, A. Ya.: Turbulent Alfven boundary layer in the polar ionosphere 1. Excitation conditions and energetics, J. Geophys. Res., 96, 19363-19374, 1991.

Tsyganenko, N. A.: Magnetospheric magnetic field model with a warped tail current sheet, Planet. Space Sci., 37, 5-20, 1989.

Walker, R. J., Erickson, K. N., Swanson, R. L., and Winckler, J. R.: Substorm-associated particle boundary at synchronous orbit, J. Geophys. Res., 81, 5541-5550, 1976.

Yago, K., Shiokawa, K., Hayashi, K., and Yumoto K.: Auroral particles associated with a substorm brightening arc, Geophys. Res. Lett., 32, L06104, doi:10.1029/2004GL021894, 2005. 